\title{
Machine Vision based Fruit Classification and Grading - A Review
}

\author{
Sapan Naik \\ Babu Madhav Institute of Information Technology \\ Uka Tarsadia University, \\ Bardoli, Surat, Gujarat, India.
}

\begin{abstract}
One of the important quality features of fruits is its appearance. Appearance not only influences their market value, the preferences and the choice of the consumer, but also their internal quality to a certain extent. Color, texture, size, shape, as well the visual flaws are generally examined to assess the outside quality of food. Manually controlling external quality control of fruit is time consuming and laborintensive. Thus for automatic external quality control of food and agricultural products, computer vision systems have been widely used in the food industry and have proved to be a scientific and powerful tool for by intensive work over decades. The use of machine and computer vision technology in the field of external quality inspection of fruit has been published based on studies carried on spatial image and / or spectral image processing and analysis. A detailed overview of the process of fruit classification and grading has been presented in this paper. Detail examination of each step is done. Some extraction methods like Speeded Up Robust Features (SURF), Histogram of Oriented Gradient (HOG) and Local Binary Pattern (LBP) are discussed with the common features of fruits like color, size, shape and texture. Machine learning algorithms like K-nearest neighbor (KNN), Support Vector Machine (SVM), Artificial Neural Networks (ANN) and Convolutional Neural Networks (CNN) are also discussed. Process, advantages, disadvantages, challenges occurring in food-classification and grading is discussed in this paper, which can give direction to researchers.
\end{abstract}

\section{General Terms}

Machine Vision, Fruit Classification, Grading.

\section{Keywords}

Fruit, Classification, Grading, Machine and Computer Vision.

\section{INTRODUCTION}

India is an agricultural country. International comparisons reveal the average yield in India is generally $30 \%-50 \%$ of the highest average yield in the world. Agriculture has comprised of $16.5 \%$ GDP by sector (2016 est.) with approximately 50\% labor force (2014 est.) and $10 \%$ total export. The budget 2017-18 pitched for more reforms in agriculture sector and increased funds for almost all areas of agriculture [1].

India exported $\$ 39$ billion worth of agricultural products in 2013, making it 7th largest agricultural exporter worldwide. According to $2010 \mathrm{FAO}$, India is world's largest producer of many fresh fruits and vegetables, milk, major species, jute, millet and castor oil seeds. India is world's second largest producer of wheat and rice. India is world's second or third largest producer of several dry fruits, agriculture based textile raw materials, roots and tuber crops, pulses, farmed fish, eggs, coconut, sugarcane and numerous vegetables [2].

Analyzing the vision is a general characteristic of our brain. Our brain takes no effort to read and understand a sign, or

\author{
Bankim Patel \\ Shrimad Rajchandra Institute of Management and \\ Computer Application, Uka Tarsadia University, \\ Bardoli, Maliba Campus, Gujarat, India.
}

separate a lion and a jaguar, or recognize people by their face. All this is too simple for humans. Where as for computers these are the actual difficulties to solve. Due to advancement in vision based computing capabilities and as algorithms can understand images and videos, systems can be prepared now which understand what we are looking at and what actions we need to perform [3]. Many machine vision algorithms are available for agricultural applications too $[4,5,6]$.

These algorithms are used frequently for speed, economic benefits and proper inspection, measurement and evaluation tasks. For acquiring a variety of information from the farms, such as fruit and vegetable detection, estimation of fruit size and weight, fruit and vegetable identification, leaf area and yield estimation, plants, classification and grading, computer vision algorithms are often used for it, autonomous Selective sprayers used and much more [7].

Among the above, fruit classification and fruit grading is one of the most important and difficult task as in the supermarket the cashiers need to know the different categories of a fruit element to determine its price [7]. In order to reduce the manual work of classification and sorting to improve the quality of the fruit grading, we can use the image processing and machine learning algorithms. Shape of the fruit, color and size can be extracted to obtain a non-destructive type of fruit classification and gradation. Machine classification and grading can be carried out automatically if some standard rules for grading criteria are made. Automatic sorting system that can perform fast, save time and reduce manual labor can be used because it has a higher priority because of the evergrowing need for high-quality fruit. Many automatic classification and sorting systems are available for various fruits such as citrus fruits, orange apples, oil palm fruits, strawberries, mangoes, lemons, dates, etc. $[8,9,10,11]$.

Parameters of non-destructive fruit classification and grading are composition, defects, size, shape, strength, flavor and color. Maturity indices for fruit grading such as flesh color, skin color and specific gravity may also be included therein (the ratio of the mango density to the density of the water)[12].

The basic steps of the automatic image-based fruit grading are: fruit image recognition, fruit object recognition, fruit classification, and finally grading by quality estimation. The parameters of the fruit grading and the weighting of each parameter are changed depending on the type of fruit. So you first have to identify the type of fruit and then decide the parameters before the grading. Fig. 1 shows the proposed model for the classification of mango fruits and their grading [11]. The same applies to all fruits. Grading standards are amended on the basis of affected persons. It is possible that the area of small Rajapuri mango can be more than the large Dashehari mango. So, before we make the grading, we need to make fruit classification and take fruits into account. 
Paper is organized as follow. Fruit classification and grading process is briefly explained in section 2. Different feature extraction techniques and machine learning algorithms are discussed in section 3 and 4 respectively. Section 5, summarize discussed literature and finally we conclude.

\section{FRUIT CLASSIFICATION AND GRADING PROCESS}

In the fruit grading process image acquisition and preprocessing, segmentation, feature extraction, knowledge base comparison and decision making steps are involved. These steps are shown in Fig. 2.

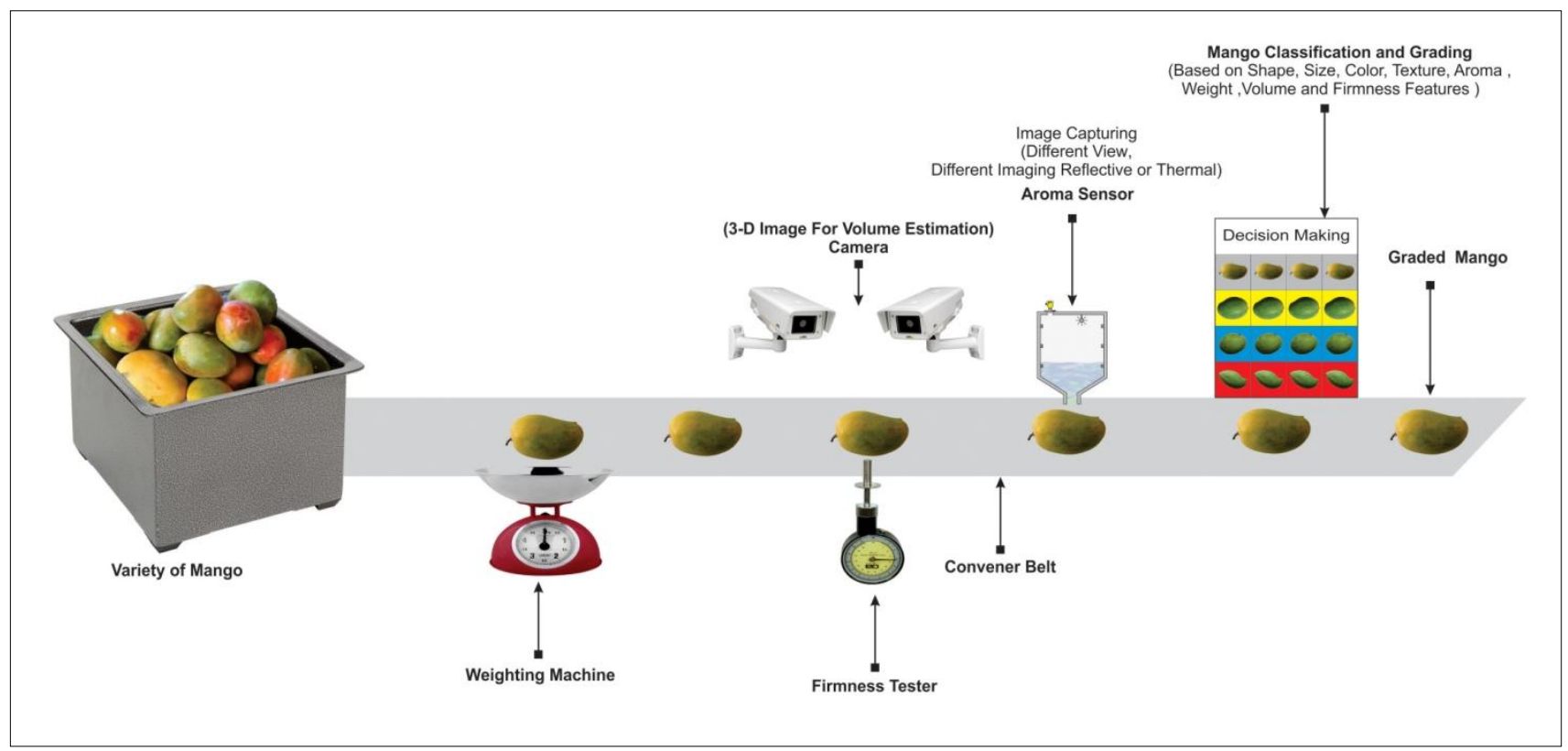

Fig 1. Proposed model for fruit classification and grading system

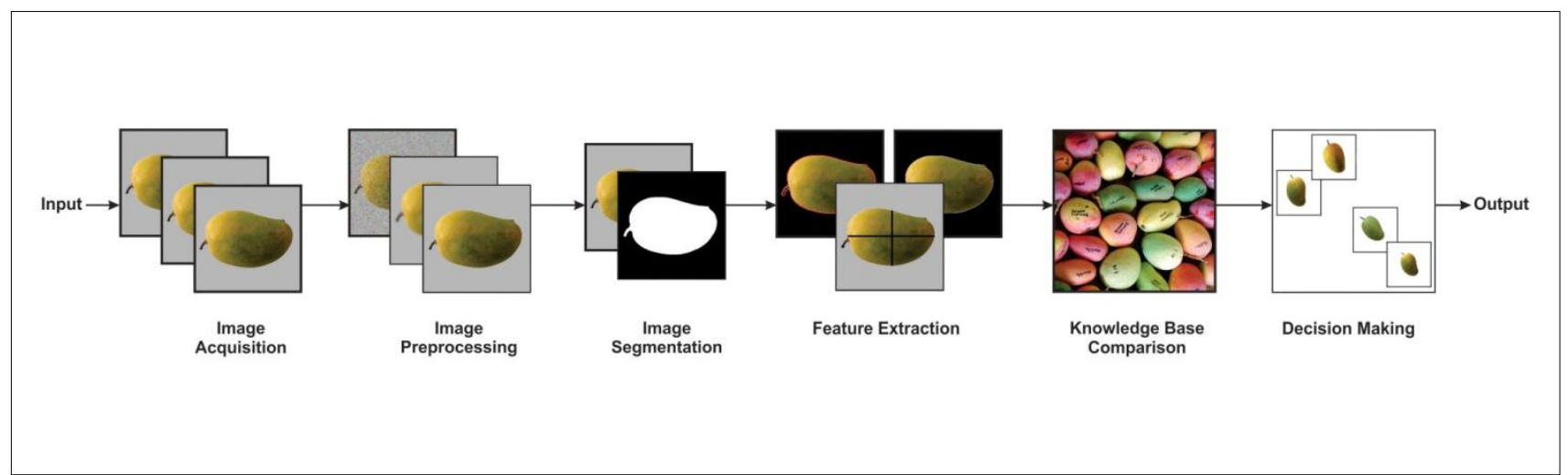

Fig 2. Flow of fruit classification and grading process

\subsection{Image Acquisition and Pre-processing}

First the image of the fruit is taken by any image capturing device. For doing so, 3 computer vision systems are most widely used in the external quality inspection of food and agricultural products, which are Traditional, Hyper spectral and Multispectral Computer Vision Systems [13]

Preprocessing methods use a small neighborhood of a pixel in an input image to obtain a new brightness value in the output image. Such preprocessing operations are also named filtration. Local preprocessing methods can be divided into the two groups according to the goal of processing: smoothing suppressed noise or other small fluctuations in the image; equivalent to the suppression of high frequencies in the frequency range. Unfortunately, the sharp edges also smoothed important information about the picture. Gradient operators are based on local derivatives of image function. Derivatives are larger at the locations of the image where the picture function changes rapidly. The goal of the gradient operators is to display such places in the picture. Gradient operators suppress low frequencies in the frequency domain (i.e., they act as a high-pass filter).

\subsection{Image Segmentation}

In image processing, image segmentation can be defined as a "process of partitioning a digital image into multiple segments" (sets of pixels, also referred to as super pixels). The goal of image segmentation is to simplify and / or change the representation of an image, which is more meaningful and easier to analyze. Image segmentation methods are 
categorized on the basis of two properties of discontinuity and similarity. Methods based on discontinuities are called boundary-based methods, and methods based on similarity are called region-based methods. The output of the segmentation is either a limitation of the object from the background or the region itself. In the color image segmentation, different color spaces such as RGB, HSI and CIELab are used [14], with the image segmentation. Some of the segmentation methods are listed in fig. 3 as follows [10].

\subsection{Feature Extraction}

Feature extraction is a low-level image processing application. For a picture, the feature is the "interest" part. In the pattern recognition literature, the name feature is frequently used to designate a descriptor. Repeatability is the desirable property of a feature detector. After image segmentation, the next step is to extract image features useful in describing fruits. Various features can be extracted from the image: color, shape, size, texture. There are some local feature detector and visual descriptor, which are used for object recognition and classification. Some of them are Speeded Up Robust Features (SURF), Histogram of Oriented Gradient (HOG) and Local Binary Pattern (LBP). All these features, feature detectors and visual descriptors are explained in next section.

\subsection{Knowledge-based comparison and decision making}

The comparison of the extracted features from the image takes place with the predetermined classification and sorting criteria

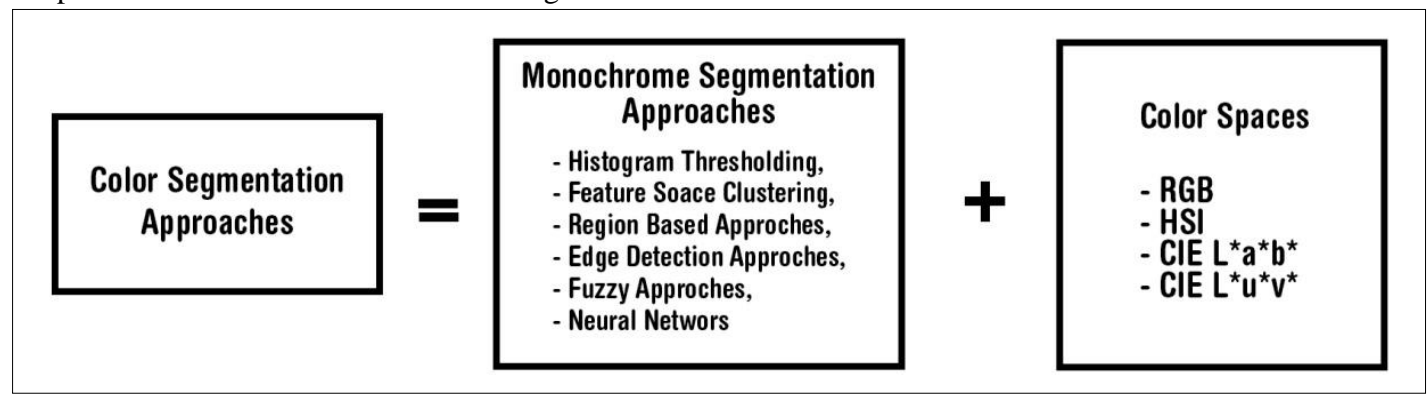

or the rules. The features are compared on the basis of the extracted features and classification is made as well as grades are given to the fruits. The knowledge-based comparison and decision-making can be done using machine learning algorithms. Some of the machine learning algorithms are Decision Tree, Regression (simple, multiple, polynomial, logistic), K-nearest neighbor (KNN), Support Vector Machine (SVM), Fuzzy Logic, Principal Component Analysis (PCA), Linear Discriminant Analysis (LDA) or Artificial Neural Networks (ANN). Some of these algorithms are explained in section 4. This is the final step of the classification and grading process. Hence all the above steps comprise the fruit classification and grading process.

\section{FEATURE EXTRACTION TECHNIQUES}

Next step in fruit classification and grading process after segmentation is feature extraction. Main and important visual external features for fruit are its color, size, shape and texture. Feature descriptor is a representation of an image or part of it, which extract useful information and discards unnecessary information. It is mainly used for image recognition and object detection. In this section, we have briefly discussed all. Some of the feature descriptors used to detect and recognize object are SURF, LBP and HOG. We have discussed these feature descriptors also here in brief.

Fig 3. Commonly used color image segmentation approaches

\subsection{Color Feature Extraction}

As color is most visually striking feature of any image it pays an important role in classification and grading system and also to identify defective fruits from normal fruits. Most of the existing system defines maturity of fruits by comparing its color with the existing predefined reference colors. Color models are divided into several models like HIS, HSV, JPG, L*a*b*, GALDA, RGB, sRGB, etc. Detail list of these color model are describe in $[15,14]$. Color feature extraction methods are widely used in agriculture applications and specifically in fruit classification and grading process. Color models like RGB, HIS and $\mathrm{L}^{*} \mathrm{a} * \mathrm{~b} *$ are used with different methods like dominant histogram, mean of color channels, etc.

Color features extraction methods broadly fall in two categories:

1. Global methods (global color histogram, histogram intersection, image bitmap)

2. Local methods (local color histogram, color correlogram, color difference histogram)

Detail description of color feature extraction is given in [16]. 2D colors histograms are used to find co-occurrence frequency and back projecting is applied to evaluate Date fruit maturity and quality in [17]. Review of different segmentation techniques, color models and feature extraction methods for fruit disease detection and fruit grading is discussed in [18]. Mango fruit sorting is performed in [19] using Gaussian Mixture Model and Fuzzy logic by considering maturity and size as parameters. $88 \%$ to $92 \%$ results are achieved for different maturity level. For orange fruit defect classification, color and texture features with novel radial basis probabilistic neural network is used and $88 \%$ accuracy is achieved in [20]. Intra class fruit classification with color and texture features is performed in [21] where ANN is used and 83-98\% accuracy is received. [22] presents fruit recognition method based on color and texture feature. For color feature extraction, some of the Python functions are available at skimage.color. Some functions are rgba2rgb(), skimage.util.invert(), label2rgb(), skimage.exposure.histogram(), rescale_intensity() and equalize_hist().

\subsection{Size feature extraction}

Fruit size is also one the most important parameter to measure the quality of fruit, larger the fruit, better it is. Larger fruits attract even more prices. It is difficult to measure fruit's size due to its natural irregularities. For size feature extraction, 
different size measures, which are most commonly used, are area, perimeter, weight, height (length), width and volume. Some other measures for size feature extraction are radius, equatorial diameter, and major and minor axes.

Applications of size inspection are one of the important parameter of fruit's quality measurement. It is explained in detail in [13]. Detailed review of non-destructive methods for fruit as well as vegetable for size determination is conducted in [23]. Weight, volume, analytical methods, asymmetrical method and statistical method are briefly explained for size estimation in [24]. For automatic examination and quality assessment of fruits and vegetables, size and volume estimation methods are described in [25]. [26] performs Date fruit classification. For the same shape and size feature with texture descriptor are used where shape and size are measured using fitting object to ellipse. Non-destructive mango fruit grading with maturity and size (area) features has been performed in [27] where thermal imaging using FLIR One camera was used. Length, maximum width, and maximum thickness are used with simple linear regression (SLR), multiple linear regression (MLR) and artificial neural network (ANN) to estimate size-mass of mango fruit in [28], where accuracy and success rate of $96.7 \%$ is achieved. Length, maximum diameter of the equatorial section, and projected area is used with stepwise multiple linear regression method to classify kiwifruit in [29]. Here accuracy reaching $98.3 \%$ by proposed method. For size feature extraction of labeled region, below is the Python function.

skimage.measure.regionprops(label_image, intensity_image $=$ None, cache $=$ True)

\subsection{Shape feature extraction}

While purchasing fruit as well as for classification and grading, shape is considered very important. The objective of the shape description is characterize the shape in such a way that the values are very similar objects in the same form class or category, and quite different for objects in different categories. This is known as the uniqueness condition. Besides the uniqueness and invariance to affine transformations, namely translation, rotation, scaling, others desirable property of any shape description method is nonambiguity or completeness [30].

Size dependent measurements of shape include compactness, elongation, convexity, roughness, etc. while size-independent measurements of shape includes region-based (statistics of pixel's spatial information) and boundary-based (Fourier transform, discrete wavelet transform, autoregressive models, etc.). Some of the shape descriptors and techniques are explained in brief in [24].

Multiple appearances with color, shape and size feature are used for identification of Strawberry cultivar and its quality evaluation in [31] where classification is increased to $68 \%$ compared to single feature. In [32], local binary pattern (LBP) or weber local descriptor (WLD) histogram with Fisher discrimination ratio (FDR) based feature selection is used for shape-size and texture based Date fruit classification and achieved 98\% classification accuracy. Color histogram, texture and shape features used with PCA, fitness-scaled chaotic artificial bee colony (FSCABC) algorithm and feed forward neural network (FNN) to classify fruits in [33]. Accuracy achieved is $89.1 \%$. [34] reviews of different fruit grading systems and parameters are discussed in detail. Fourier-descriptor is used for shape feature extraction with SVM to classify mangoes and has achieved almost $100 \%$ classification results in [35]. Shape, texture and color (HSV) feature extraction is used to classify 20 categories of fruits in [36]. Color, shape, and texture feature extracted with PCA, biogeography-based optimization (BBO) and feed forward neural network (FNN) to classify 18 categories of fruits in [37] which achieves $89.11 \%$ accuracy. Naive Bayes is used for apple fruit classification in [38] with $91 \%$ accuracy. For computing shape index, Python function is

skimage.feature.shape_index(image, sigma $=1$, mode $=$ 'consta $n t^{\prime}$, cval=0).

\subsection{Texture Feature Extraction}

Set of two-dimensional array calculated to quantify visual texture of an image is called image texture. It provides details about the spatial organization of color or intensities in an image (region of an image). Traditional fruit recognition algorithms either ignore the texture feature of fruits, or use texture features that couldn't represent better texture of fruit images. Fruits recognition based on color and texture features are available in literature. To analyze an image texture in computer graphics, there are two approaches: Structured Approach and Statistical Approach.

Texture descriptor is used in [32] to perform Date fruit classification. Texture and shape features used with PCA to classify fruits in [39] with Neural Network as classifier. To classify 20 categories of fruits in [40], texture feature is used and to classify 18 categories of fruits in [41] which achieves $89.11 \%$ accuracy, texture feature with Neural Network is used. Color and texture features with neural network are used for orange fruit defect classification, and it has achieved $88 \%$ accuracy in [20]. As explained before fruit classification with color and texture features is performed in [21] where ANN is used and $83-98 \%$ accuracy is received. Fruit recognition based on texture feature is available in [22]. LBP or HOG can be used for texture features extraction.

\subsection{Speeded Up Robust Features (SURF)}

SURF is specific algorithm used for feature detector and feature descriptor. It is partially inspired by the Scale Invariant Feature Transform (SIFT) descriptor. Due to its faster detection feature now-a-days SURF is used more than SIFT. Mainly, SURF algorithm is used in object recognition, image registration, and classification or $3 \mathrm{D}$ reconstruction applications.

To detect interest points, SURF uses an integer approximation of the determinant of Hessian blob detector. Its feature descriptor is based on the sum of the Haar wavelet response around the point of interest. For object recognition task, SURF algorithm is used because of its powerful attributes, including scale invariance, translation invariance, lighting invariance, contrast invariance, and rotation invariance $\&$ it can detect objects in images taken under different extrinsic and intrinsic settings.

The algorithm has three main parts [42]:

1. Interest/key point detection - based on the approximate Hessian matrix.

2. Feature Description - every neighbor region around each point, which should be as discriminative as possible.

3. Feature Matching - matched based on some distance calculations. 
SURF based bag-of-features and spatial pyramid matching kernel approaches are used to recognize food items in [43] and $86 \%$ classification rate is achieved. Texture analysis is performed with SVM classifier in [44] where $85 \%$ for pineapples and $100 \%$ for bitter melons result is achieved. SURF based Fruit defect detection is performed in [45]. Below is sample Python script for extracting SURF.

img $=$ cv2.imread('fruit.png',0)

\# Here Hessian Threshold set to 400

surf $=c v 2 . S U R F(400)$

\# Find keypoints and descriptors directly

$k p$, des $=$ surf.detectAndCompute(img,None $)$

\subsection{Histogram of Oriented Gradients(HOG)}

HOG is a feature descriptor used for object detection in computer vision, which counts frequencies of oriented gradients in localized part of an image. HOG is good feature descriptor in the sense that it fetches oriented gradient and the magnitude of gradients is high at corner and edges where intensity changes and edges represents shape of object [46]. For finding HOG feature, first $[-1,0,1]$ filter is applied in horizontal as well as vertical direction. Next find magnitude and direction of gradient using formulas. Next image is divided in $8 \times 8$ cells and Histogram of gradient is calculated and finally HOG feature vector is generated.

In the field of agriculture, HOG is used for object detection and recognition. HOG with Zernike Moments is used for plant leaf classification in [47]. Here SVM is used as classifier and experiments confirmed on datasets of the Flavia and the Swedish Leaves. HOG feature with SVM is used, to get Nutritional Information by Food Classification in [48]. Situ Leaf Classification is performed using HOG and multi-feature probabilistic NN classifier in [49], which provides $86.07 \%$ accuracy. Find sample Python function below.

skimage.feature.hog(image, orientations $=9$, pixels_per_cell $=($ 8, 8),cells_per_block $=(3,3)$, block_norm $=$ 'L1', visualise $=$ Fal se,transform_sqrt=False, feature_vector $=$ True)

\subsection{Local Binary Patterns (LBP)}

LBP is used for classification problem and it is a visual/texture descriptor, which works on texture/pattern in an image. It can be used for texture classification. It gets popular after work get published by Ojala et al. Multi resolution Grayscale and Rotation Invariant Texture Classification with Local Binary Patterns, 2002. LBP uses texture's local representation, which is comparing each pixel with its surrounding neighbor pixels. If LBP has maximum two 01 or 1-0 transitions, it is considered to be uniform [50]. Detailed working of LBP is discussed in [50].

LBP is used for fruit classification and grading too. New Opponent Color Local Binary Patterns is proposed in [51] which provides equivalent result as convolutional neural network's last generated features and performs far better than basic LBP for color texture classification. The color completed local binary pattern merged with a HSV color histogram and a Border/interior pixel classification color histogram on 60 different fruits and vegetables can achieve $94.26 \%$ recognition rate in [52]. Local binary pattern and Weber local descriptor histogram for texture recognition is used in [32] for Date fruit classification. Below is Python function for LBP.

skimage.feature.local_binary_pattern(image, $P, R$, method='d efault').

\section{MACHINE LEARNING ALGORITHMS}

As the last phase of fruit classification and grading process is knowledge-based comparison and decision making, machine learning algorithms play very important role as classifier and decision makers. In below section, we have briefly described $\mathrm{K}-\mathrm{NN}$, SVM, ANN and CNN.

\subsection{K- nearest neighbor ( $\mathrm{KNN})$}

$\mathrm{K}-\mathrm{NN}$ focuses on similarity (proximity) of samples measured by a distance metric and are a statistical classifier. Its job is to assign data to the most represented category within its closest $\mathrm{k}$ neighbors'.

To perform K-NN firstly we select number $\mathrm{K}$ of neighbor, secondly based on Euclidian distance we select $\mathrm{k}$ nearest neighbors of new point, lastly number of data point in each category are counted and then a new point is chosen where you find more points. Fig. 4 demonstrates the working of KNN. Below is sample Python script for implementing K-NN.

\# Fitting K-NN to the Training set

from sklearn.neighbors import KNeighborsClassifier

classifier $=$ KNeighborsClassifier(n_neighbors $=5$, metric $=$ 'minkowski', $p=2$ )

classifier.fit(X_train, $y_{-}$train)

\# Predicting the Test set results

y_pred $=$ classifier.predict $\left(X \_t e s t\right)$

In [54], fruit recognition system is proposed which uses the KNN algorithm as a classifier to classify fruit based on mean color values, shape roundness value, area and perimeter values of the fruit (represent size). For K-NN, value of $\mathrm{K}$ is chosen 1 and used Euclidean distance.

\subsection{Support Vector Machine (SVM)}

One of the powerful classification algorithms that have shown state of-the-art performance in different varieties of classification tasks is SVM. Classification of both linear and nonlinear data is done using a new method by SVM. Using kernel functions, SVM nonlinearly maps data to a highdimensional space. 


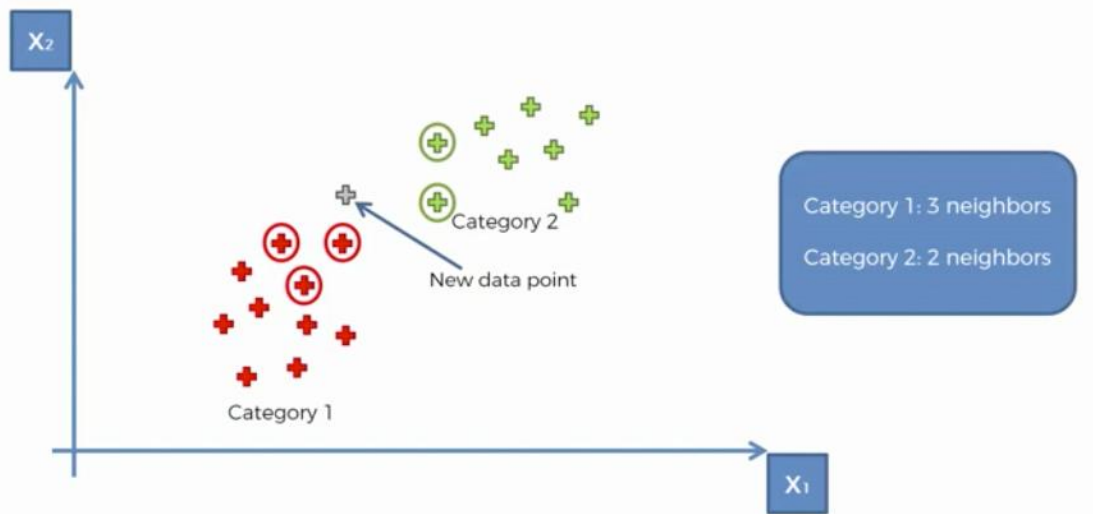

Fig 4. K-NN intuition for $K=5[53]$

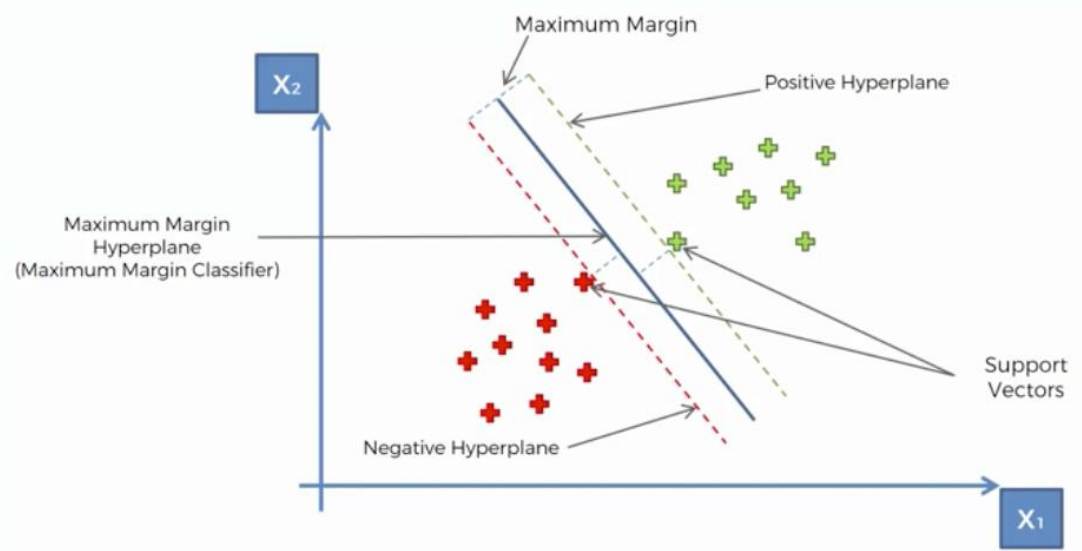

Fig 5. SVM intuition [53]

In that high dimensional space it tries to find the linear optimal hyper plane that separates data with maximum margin. SVM was proposed for only 2-class problems; in multi-class problem SVM is extended using near-against-one or one-against-all strategies.

SVM tries to draw a hyper plane between to classes such that the distance between support vectors can be maximized. SVM support vectors, which are nothing but extreme points of both classes that is the reason why SVM is considered special. Basic concepts of SVM working are shown in below fig. 5 .

Sample Python script for making SVM is shown below.

\section{\# Fitting SVM to the Training set}

from sklearn.svm import SVC

classifier $=$ SVC $($ kernel $=$ 'linear', random_state $=0)$

classifier.fit(X_train, $y_{\text {_train }}$ )

\# Predicting the Test set results

y_pred $=$ classifier.predict $\left(X \_t e s t\right)$

Image classification for detection of winter grapevine buds in natural conditions is performed using scale-invariant features transform, bag of features and support vector machines in [55]. To identify weather the particular area needs to be sprayed or not SVM is used as decision maker (weed identification process) in [56]. Browning degree on mango fruit is automatically detected using LS-SVM in [57]. Different brands of sesame oil are classified using Support Vector Machine-Multiclass Forward Feature Selection (SVMMFFS), Successive Projection Analysis (SPA) and Uninformative Variable Elimination (UVE) algorithms in [58]. Color moments, GLCM, and Wavelets energy and entropy is used for color and texture feature extraction in [59] for tomato grading in two classes. PCA and SVM are used for dimensionality reduction and as a classifier respectively. 92\% accuracy is achieved.

\subsection{Artificial Neural Network (ANN)}

Computer programs which are biologically inspired are designed to simulate the way in which the human brain processes information is known as ANN. ANNs are trained computer programs, which works similar to our brains. An extension of many classification techniques can be regarded as artificial neural networks. ANNs are robust in dealing with the ambiguous data and the kind of problems that require the interpolation of large amounts of data. 


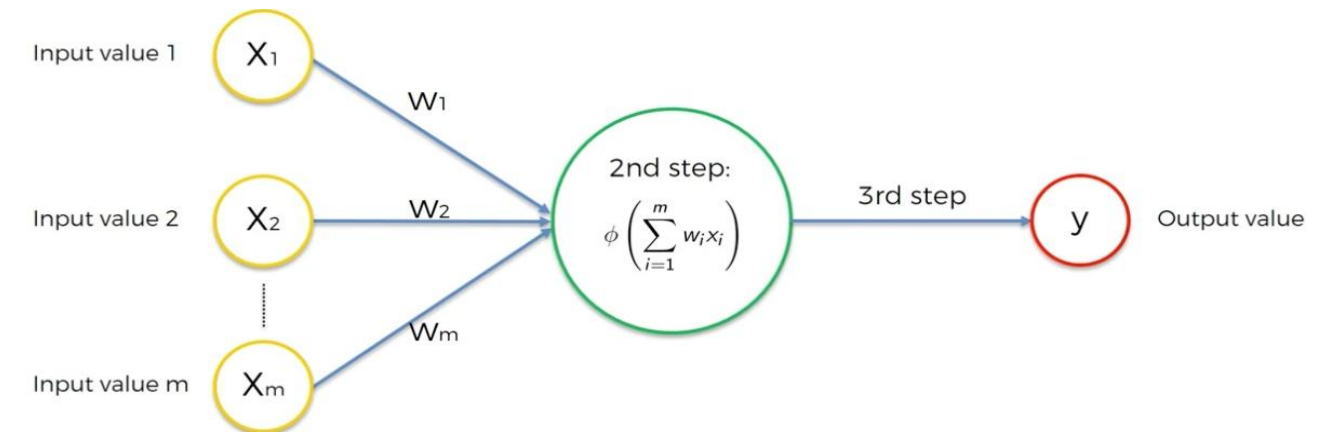

Fig 6. Basic structure of ANN [53]

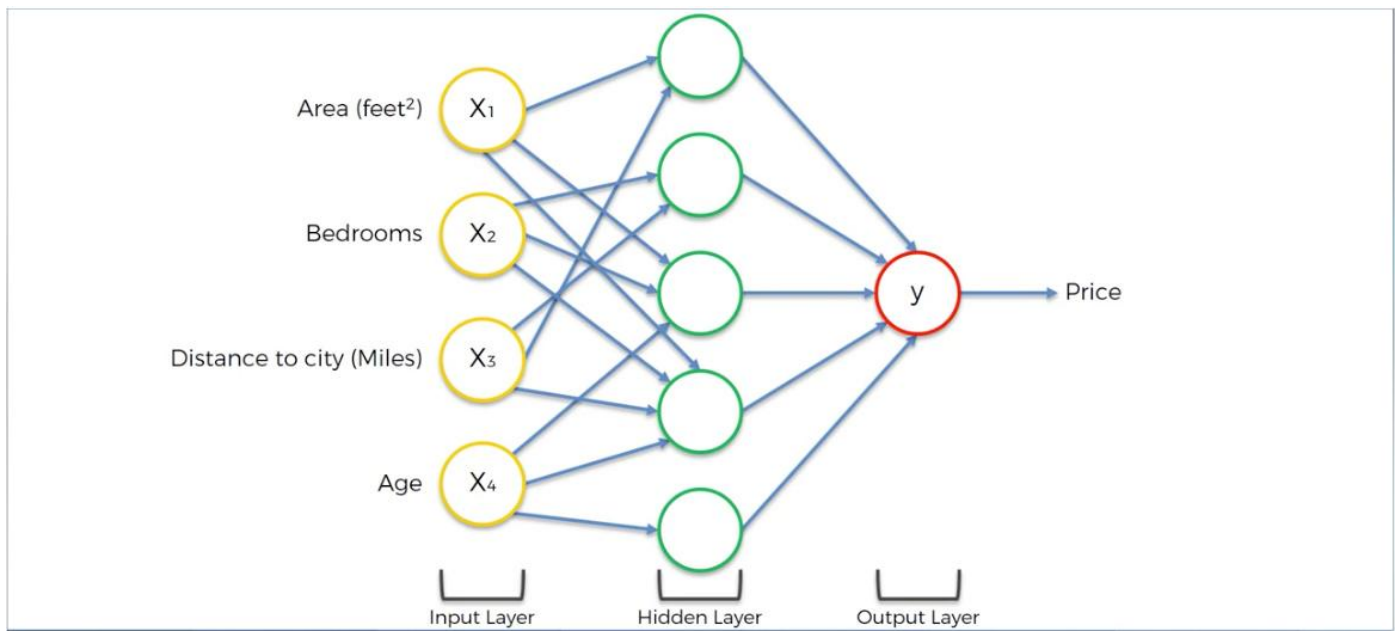

Fig 7. ANN layers and neurons intuition [53]

Three layers are contained by ANN, which are, input, hidden and output. Nodes also known as neurons are present in each layer. Activation function is in hidden layer and output layer. Based on application in hand activation functions are chosen. Some of the activation functions are rectifier, sigmoid, hyperbolic tangent. Link and link contains weights connects nodes with each other. Below fig. 6 and 7 shows sample ANN structure and layers connections.

The working of ANN is in two phases namely training and testing. During training phase one need to provide input and corresponding output. Using back propagation and cost function value of weights gets set in training phase. As soon as training gets over, ANN is ready for test. Below fig. 8 show sample training and back propagation.

ANNs easily solve complex problems. Much more processing power than other algorithms are required by ANN. There are many types of ANN like Feed Forward Network, Single Layer Perceptron, Multi-Layer Perceptron, Recurrent Neural Network, Self-organizing Map and other [60]. Sample Python script for making ANN is given below.

\# Importing the Keras libraries and packages
import keras
from keras.models import Sequential
from keras.layers import Dense
\# Initialising the ANN
classifier = Sequential()
\# Adding the input layer and the first hidden layer
classifier.add(Dense(output_dim = 6, init = 'uniform',
activation = 'relu', input_dim =11))

\# Adding the second hidden layer classifier.add(Dense(output_dim $=6$, init $=$ 'uniform', activation $=$ 'relu'))

\# Adding the output layer

classifier.add(Dense(output_dim $=1$, init $=$ 'uniform', activation = 'sigmoid'))

\# Compiling the ANN

classifier.compile(optimizer = 'adam', loss=

'binary_crossentropy', metrics $=[$ 'accuracy'])

\# Fitting the ANN to the Training set

classifier.fit(X_train, y_train, batch_size $=10$, nb_epoch $=$ 100)

In [61] fruit disease identification is performed where $\mathrm{K}$ means clustering is applied for image segmentation, color, morphology, texture and structure features are used with ANN. Sort tomato fruits using shape, texture and color features. Circularity, size, maturity, and defects are used as parameter. Probabilistic Neural Network (PNN) is used as classifier and $84.4 \%$ accuracy is achieved for defect detection in [62]. 10 different vegetables are recognized using the color histogram and statistical texture features with neural network as a classifier in [63]. Results of $96.55 \%$ accuracy is gained. In [64], back propagation artificial neural network with two hidden layers are used to predict the texture characteristics from food surface. Fruit classification using computer vision and feed forward neural network is proposed [66]. Fruit detects are classified using color and texture features with gray level co-occurrence matrix and Radial Basis Probabilistic Neural Networks in [65]. 389 out of 400 fruits are accurately classified. 


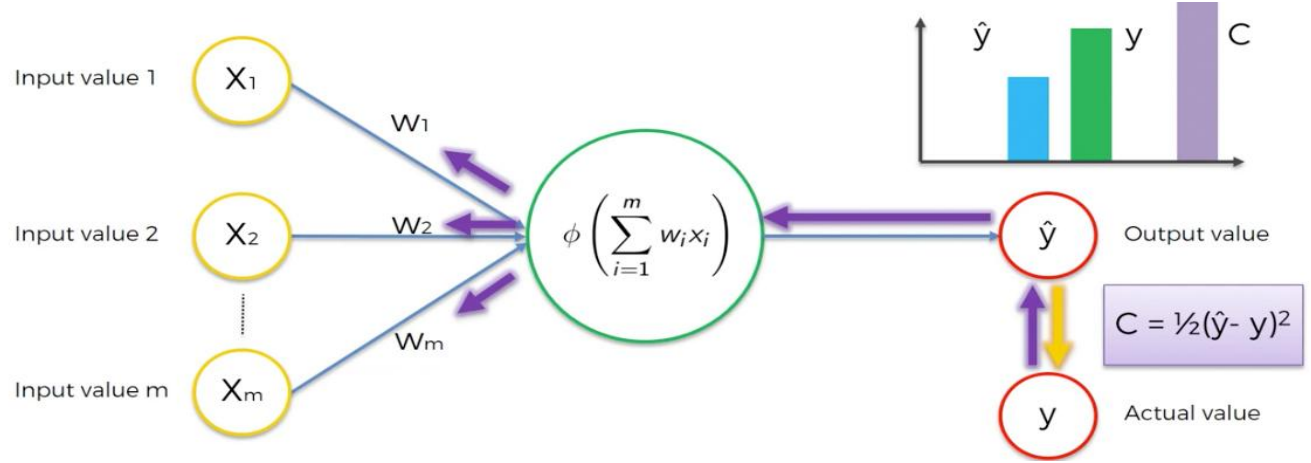

Fig 8. Learning process of ANN [53].

\subsection{Deep Learning / Convolutional Neural Networks}

For the image classification and recognition tasks, development in deep learning and convolution neural network (CNN) have been proved to be a bonus, including for the problem of fruit recognition. Deep learning learns automatically, the features of images. It extracts global features and contextual details, which reduces error drastically in image recognition [67]. Recently the deep learning received more popularity than any other machine learning algorithms as shown in below fig. 9 [68]. However, the associated research in fruit classification using this method is less presently.

Deep learning received main attention when Hinton's team got the champion of the ImageNet image classification [73]. Edge features and texture features to identify local as well as global features are used in it. Many researchers have made good accomplishments in the field of image recognition using this automatic learning features technique [39].

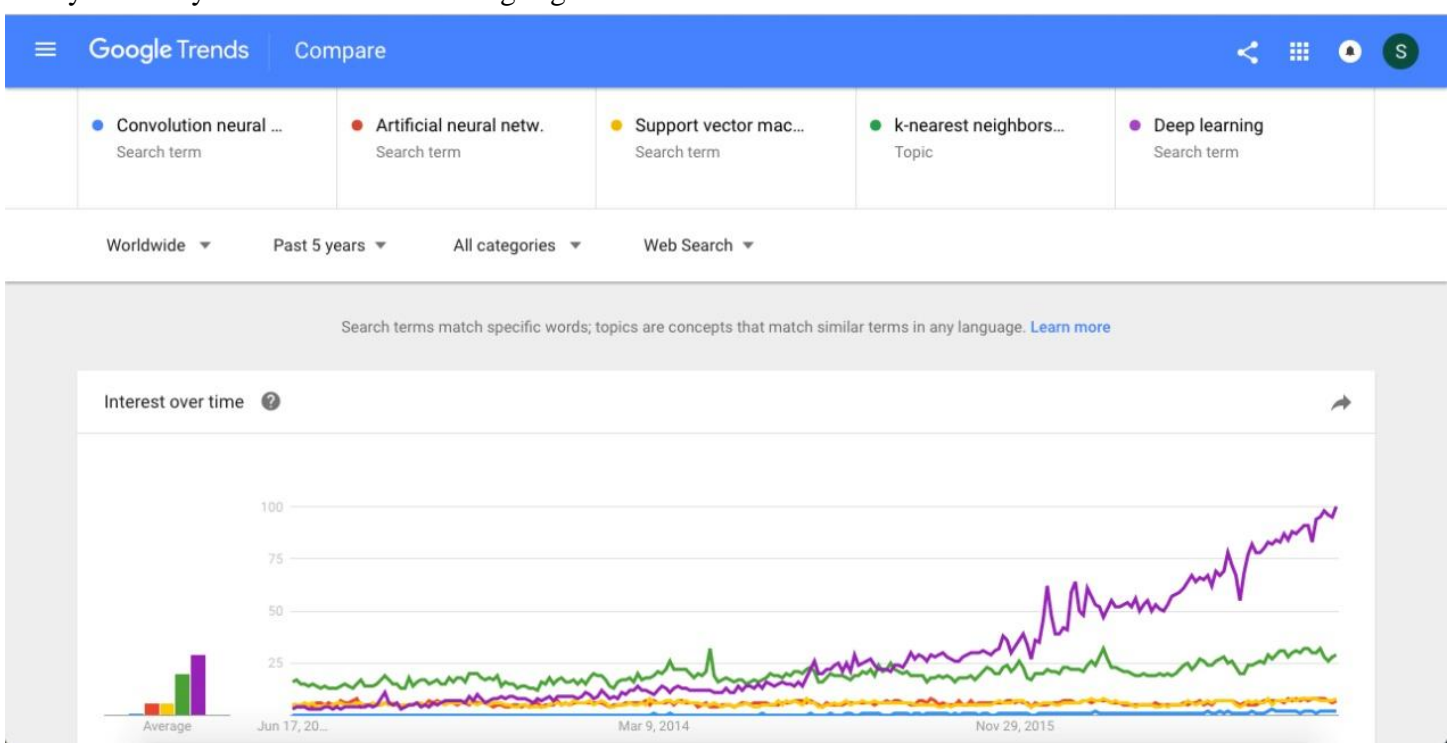

Fig 9. Recent trends of machine learning algorithms [68]

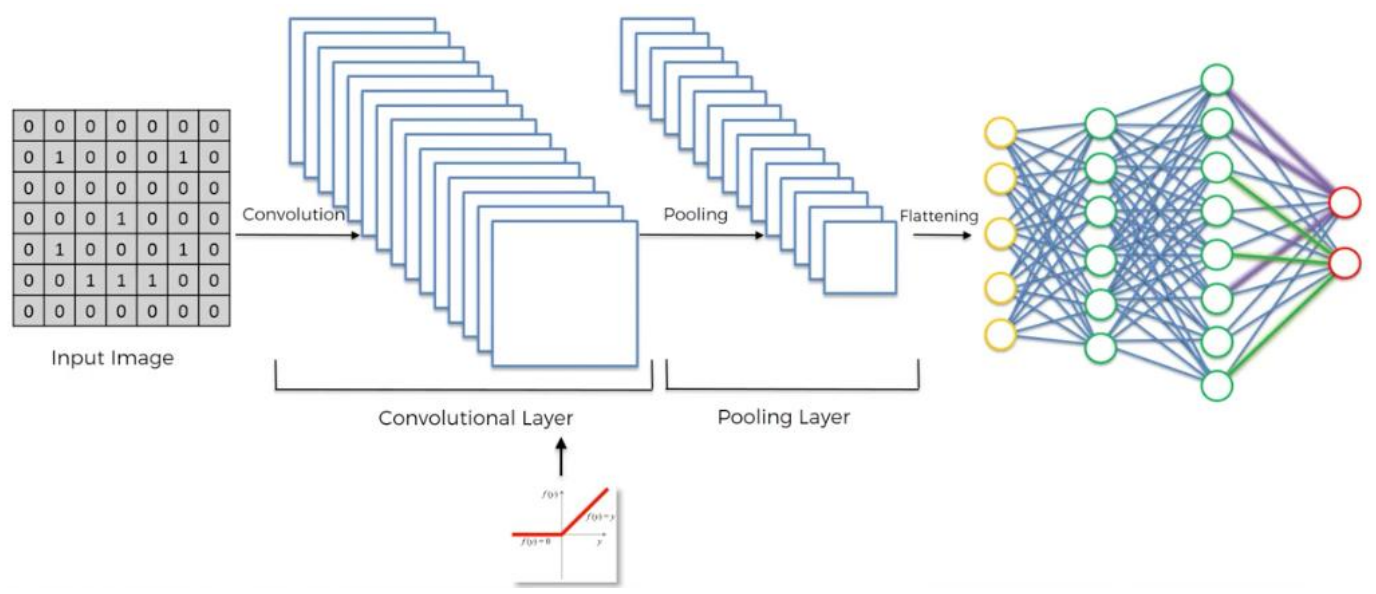

Fig 10. Convolutional Neural Network intuition [53] 
QuocNet, AlexNet, Inception (GoogLeNet), BN-Inception-v2 are some models proposed later and showed good results. Google trained random 10 million images with neural network of 9 layers and classification performed on ImageNet data set of 2000 categories, $70 \%$ improved results are achieved than the previous best results [69]. Identification of weed based on $\mathrm{K}$-means feature learning combined with convolutional neural network is done [70]. PASCAL-VOC - the state-of-the-art detection framework [40] consists of two stages. DeepFruits: A fruit detection system using deep neural networks is proposed in [41] and the model is retrained to perform the detection of seven fruits, with the entire process taking four hours to annotate and train the new model per fruit. Google has released code for running image recognition in their latest model, Inception-v3. Weed identification based on K-means feature learning combined with convolutional neural network is performed in [71].
To train CNN, we simply need to provide input image and associated label. We do not need to extract any features. CNN do that for us automatically. CNN mainly contain four steps namely Convolution, Pooling, Flattening and Full Connection as shown in fig. 10 [53].

In Convolution step, feature detector (Kernel/Filter) is applied on input image and Feature Map is created. Many such feature detectors are applied on input image and as many Feature Maps are generated on Convolution Layer (In convolution step). Some basic feature detectors are Sharpen, Edge Detect, Edge Enhance, Median, and Emboss. In same step, next is ReLU layer comes in which our all Feature Maps are passed through Rectifier function. It normally used to break linearity in the image. In ReLU layer, instead of Rectifier function one can use other functions like Sigmoid or Leaky ReLU too if required.

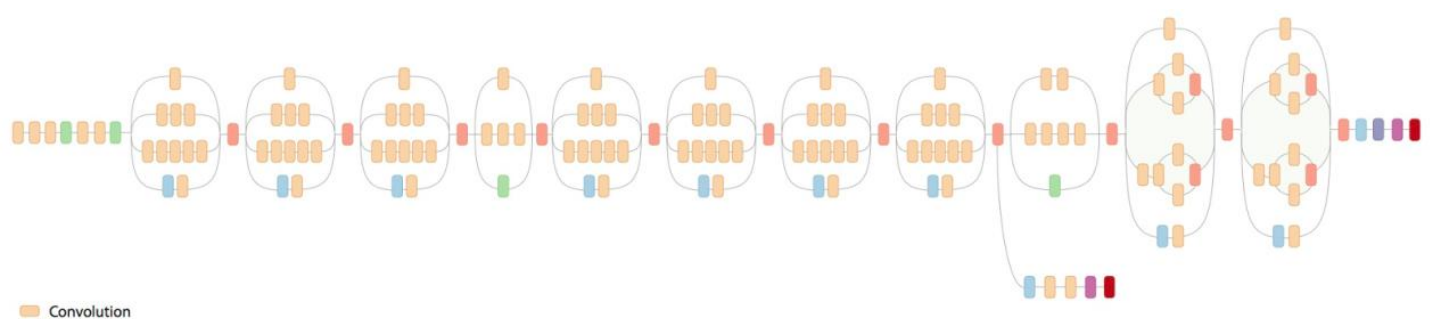

Fig 11. Inception-v3 CNN visualization [72]

In Pooling step, all feature maps are given as input and pooled feature map is received as output. Pooling function can be any like min, max or median. Normally images can be rotated, scaled or with different backgrounds. Take example of Lion in one image. Lion on other image can be small, big, tilted or with different background, different color condition. So pooling is helpful in mapping the right and needed features of the images, which can be used to map same object in other image. In our example we can map Lion in all images even though it is titled or small or big.

In next Flattening step, we simply convert pooled feature map into single vector because it will next given as input to artificial neural network. Last step is full connection, which is nothing but implementing artificial neural network where all nodes of input, hidden and output layers are connected with each other. Below image summarize all the steps of CNN.

We can have more convolution layers and pooling layers based on our application in hand and our requirements. For example, Inception-v3 can be visualized with following schematic diagram of fig. 11

GPU is preferable for $\mathrm{CNN}$ as it requires lots of processing power but one can use CPU also for small applications. Below is sample Python script for creating CNN.

\# Importing the Keras libraries and packages

from keras.models import Sequential

from keras.layers import Convolution2D

from keras.layers import MaxPooling2D

from keras.layers import Flatten

from keras.layers import Dense

\# Initialising the CNN

classifier $=$ Sequential ()

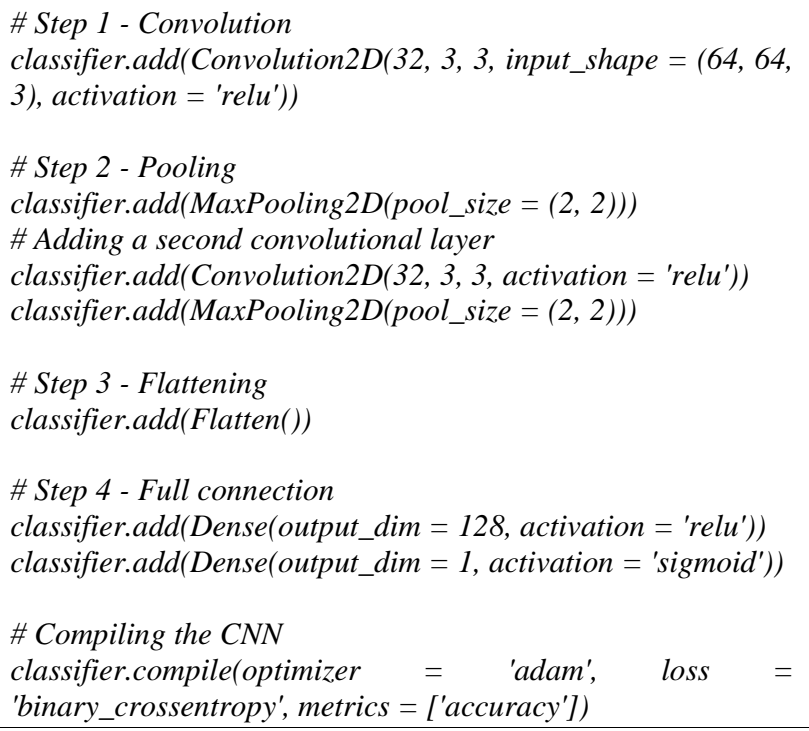

Other classifiers are also available. Comparison of different machine learning algorithms with their advantages and disadvantages has been discussed in [24].

\section{SUMMERIZATION}

Table 1 summaries the recent fruit classification and grading methods. It also summaries some of the literature reviews for fruit classification and grading or for some specific feature used in it. 


\section{CONCLUSION}

External properties of fruits like color, size, shape, texture and different defects are very important attributes of fruits for classification and grading. Now a days due to advancement in machine vision and availability of low cost hardware and software, manual work of fruit classification and grading has been replaced with automated machine vision systems. Other reason of non-destructive automation can be its ability to produce accurate, rapid, objective and efficient results over manual work.

This paper reviews the basic process flow of fruit classification and grading. Feature extraction methods for color, size, shape and texture are explained with SURF, HOG and LBP features. Finally some machine learning approaches like K-NN, SVM, ANN and CNN are briefly discussed. Though some challenges are still need to overcome, but machine vision will prove to be the future for non-destructive fruit classification and grading.

In future, we can work on image classification for local fruits and vegetables. We can also prepare algorithms and machines for fruits and vegetable grading. It can also be used for plants/ leaves/ flowers identification and classification. A system can be develop which will identify plant/leaf/flower and provide information regarding it. We can also work on some more features for grading and classification, which can identify types of disease and/or texture structure of fruits. All these are future direction. One can work on these and prepare prototype model, which can be used in industries. One can even develop mobile applications for the same based on above methods and farmers or general public can use it for identification, classification and grading of horticultural products.

Table 1. Summary of recent fruit classification and grading methods

\begin{tabular}{|c|c|c|c|c|}
\hline Fruit & Features & Classifier & Accuracy (\%) & Reference \\
\hline \multirow{3}{*}{ Date } & Color, Texture & $\begin{array}{l}\text { Nearest Neighbor, } \\
\text { DA, ANN }\end{array}$ & 83-98 & S. Jana et al., 2016 \\
\hline & Size, Shape, Texture & LBP, WLD, FDR & 98 & G. Muhammad, 2015 \\
\hline & Color & $\mathrm{BP}$ & & D. Zhang et al.,2014 \\
\hline \multirow{5}{*}{ Mango } & Maturity, Size & $\begin{array}{c}\text { Fuzzy, } \\
\text { Thermal Imaging }\end{array}$ & 90 & S. Naik et al., 2017 \\
\hline & Size, Volume & MLR, ANN & 96.7 & K. Utai et al., 2015 \\
\hline & Shape, Weight & $\begin{array}{c}\text { FD, } \\
\text { DA/SVM/Weight }\end{array}$ & $98.3 / 100 / 95$ & F.S.A. Sa'ad et al., 2015 \\
\hline & Color, Size & GMM & $88.3-90.5$ & C. S. Nandi et al.,2014 \\
\hline & $\begin{array}{l}\text { Color, fractal } \\
\text { analysis }\end{array}$ & LS-SVM & Up to 100 & H. Zheng, 2012 \\
\hline \multirow[b]{2}{*}{ Tomato } & Color, texture & PCA, SVM & 92 & N.A. Semary et al.,2015 \\
\hline & $\begin{array}{l}\text { Color, Shape, } \\
\text { Texture }\end{array}$ & PNN & 84.4 & O.O. Arjenaki, 2013 \\
\hline Grapevine buds & SIFT, Bag of Feature & $\begin{array}{c}\text { SVM } \\
\text { (Detection } \\
\text { /Classification) }\end{array}$ & $97.7 / 86$ & D. S. Pérez et al., 2017 \\
\hline Orange & Color, Texture & ANN & 88 & G. Capizzi et al.,2016 \\
\hline Apple & Color, Size & Naive Bayes & 91 & M. Ronald et al., 2016 \\
\hline Kiwi & Shape & MLR & 98.3 & L. Fu et al., 2016 \\
\hline Strawberry & Color, Size, Shape & $\begin{array}{c}\text { Cluster Analysis, } \\
\text { Multidimensional scaling, DA } \\
\end{array}$ & $>68$ & K. Yamamoto et al., 2015 \\
\hline \multirow{8}{*}{ Mix } & Color, shape, texture & PCA, BBO, FNN & 89.11 & Y. Zhang et al., 2016 \\
\hline & $\begin{array}{l}\text { Color(HSV), shape, } \\
\text { texture }\end{array}$ & & & F. Garcia et al., 2016 \\
\hline & Color, texture & ANN & & A. Awate et al., 2016 \\
\hline & Color, Texture & Co-occurrence matrix, RBPNN & 97.25 & G. Capizzi et al.,2015 \\
\hline & $\begin{array}{c}\text { Color, Shape, } \\
\text { Texture }\end{array}$ & $\begin{array}{c}\text { PCA, } \\
\text { FSCABC, FNN }\end{array}$ & 89.1 & Y. Zhang et al., 2014 \\
\hline & Color, Texture & ANN & 96.55 & M. T. Chowdhury, 2013 \\
\hline & Texture & BPNN & $96-98$ & Fan et al., 2013 \\
\hline & Color, Texture & & 98 & Vishwanath.B.C et al., 2012 \\
\hline \multirow{8}{*}{ Review } & \multicolumn{3}{|l|}{ Color, Size, Shape } & S. Khoje, 2015 \\
\hline & \multicolumn{3}{|l|}{ Color } & Srivastava et al., 2015 \\
\hline & \multicolumn{3}{|c|}{ Color, Disease detection, fruit grading } & U. Solanki et al.,2015 \\
\hline & \multicolumn{3}{|c|}{ Color, Size, Shape, Texture, Surface defect } & B. Zhang et al.,2014 \\
\hline & \multicolumn{3}{|c|}{ Color, Size, Shape, KNN, SVM, ANN, Fuzzy } & R. Pandey, 2014 \\
\hline & \multicolumn{3}{|l|}{ Shape } & Moreda, G. P., et al., 2012 \\
\hline & \multicolumn{3}{|c|}{ Color, Size, Shape, Texture, Volume, Defects and Internal parameter } & S. Cubero et al., 2011 \\
\hline & \multicolumn{3}{|l|}{ Size } & G.P. Moreda et al., 2009 \\
\hline
\end{tabular}




\begin{tabular}{|l|l|}
\hline ANN - Artificial neural network & GMM - Gaussian Mixture Model \\
\hline BBO - Biogeography-based optimization & LBP - Local binary pattern \\
\hline BP - Back projection & MLR - Multiple linear regression \\
\hline BPNN - Back Propagation Neural Network & PCA - Principal Component Analysis \\
\hline DA - Discriminant Analysis & PNN - Probabilistic neural network \\
\hline FD - Fourier-descriptor & RBPNN - Radial Basis Probabilistic Neural Networks \\
\hline FDR - Fisher discrimination ratio & SVM - Support Vector Machine \\
\hline FNN - Feed forward neural network & WLD - Weber local descriptor \\
\hline FSCABC - fitness-scaled chaotic artificial bee colony algorithm \\
\hline
\end{tabular}

\section{ACKNOWLEDGMENT}

Authors gratefully acknowledge the Uka Tarsadia University for financial assistance under research promotion scheme (UTU/RPS/1404(5-5)/2014-15). Authors would like to thank Mr. Yash Rana, Mr. Vishal Zaveri and Ms. Bushra Ansari for making of illustrative figures and searching content.

\section{REFERENCES}

[1] Agriculture in India: Information About Indian Agriculture \& Its Importance. [online] https://www.ibef.org/industry/agriculture-india.aspx. Date: 18.06.2017.

[2] Agriculture in India [online] https://en.m.wikipedia.org/wiki/Agriculture_in_India. Date:18.06.2017.

[3] Google I/O https://events.google.com/io/. Date:18.06.2017.

[4] Gomes et al., "Applications of computer vision techniques in the agriculture and food industry: a review", Eur. Food Res. Technol., 235 (6), 989-1000. 2012.

[5] Zhang et al., "Application of computer vision technology in agricultural field", Applied Mechanics and Materials, vol. 462. Trans Tech Publ, pp. 72-76, 2014.

[6] Vibhute et al., "Applications of image processing in agriculture: a survey", Int. J. Comput. Appl., 52 (2), 3440, 2012.

[7] Diego Sebastián Pérez et al., "Image classification for detection of winter grapevine buds in natural conditions using scale-invariant features transform, bag of features and support vector machines", Computers and Electronics in Agriculture 135, 81-95, January 2017.

[8] A. Vyas et al, "Colour Feature Extraction Techniques of Fruits: A Survey", International Journal of Computer Applications (0975 - 8887) Volume 83 - No 15, December 2013.

[9] S. Naik et al, "Shape, size and maturity features extraction with fuzzy classifier for non-destructive mango (Mangifera Indica L., cv. Kesar) grading", TIAR(978-1-4799-7758-1), 5-11, July 2015.

[10] Rashmi Pandey et al.," Image Processing and Machine Learning for Automated Fruit Grading System: A Technical Review", International Journal of Computer Applications (0975 - 8887) Volume 81 - No 16, November 2013.

[11] Sapan Naik and Bankim Patel, "Usage of Image Processing and Machine Learning Techniques in Agriculture - Fruit Sorting", CSI Communications, ISSN
0970-647X, Volume No. 37, Issue No. 7, pp. 25-27, October 2013.

[12] D.C. Slaughter, "Nondestructive maturity assessment methods for Mango: A review of literature and identification of future research needs", January 2009.

[13] Baohua Zhang et al.," Principles, developments and applications of computer vision for external quality inspection of fruits and vegetables: A review", Food Research International, 62, 326-343, 2014.

[14] Sapan Naik and Bankim Patel," CIELab based color feature extraction for maturity level grading of Mango (Mangifera Indica L.)", National journal of system and information technology (0974-3308), VOLUME 7, NO. 1, June 2014.

[15] Cubero, S., Aleixos, N., Moltó, E. et al., "Advances in Machine Vision Applications for Automatic Inspection and Quality Evaluation of Fruits and Vegetables", Food Bioprocess Technol, 4: 487. doi:10.1007/s11947-0100411-8, 2011

[16] Srivastava, Divya, Rajesh Wadhvani, and Manasi Gyanchandani. "A Review: Color Feature Extraction Methods for Content Based Image Retrieval." International Journal of Computational Engineering \& Management 18.3 : 9-13, 2015.

[17] Dong Zhang et al.,"Date maturity and quality evaluation using color distribution analysis and back projection", Journal of Food Engineering, Volume 131, Pages 161169, June 2014.

[18] Uravashi Solanki et al., "A Survey on Detection of Disease and Fruit Grading", International Journal of Innovative and Emerging Research in Engineering Volume 2, Issue 2, 2015.

[19] C. S. Nandi et al., "Machine Vision Based Techniques for Automatic Mango Fruit Sorting and Grading Based on Maturity Level and Size", DOI: 10.1007/978-3-31902315-1_2, Springer International Publishing Switzerland 2014.

[20] Giacomo Capizzi et al., "A Novel Neural NetworksBased Texture Image Processing Algorithm for Orange Defects Classification", IJCSA, 2016.

[21] Jana, Susovan, and Ranjan Parekh. "Intra-class Recognition of Fruits using Color and Texture Features with Neural Classifiers." International Journal of Computer Applications 148.11, 2016.

[22] Dr Vishwanath.B.C, S.A.Madival, Sharanbasava.Madole, "Recognition of Fruits in Fruits Salad Based on Color and Texture Features", International Journal of Engineering Research \& Technology, Vol.1 - Issue 7, September, 2012. 
[23] G.P. Moreda et al, "Non-destructive technologies for fruit and vegetable size determination - a review", Journal of Food Engineering 92, 119-136, 2009.

[24] Rashmi Pandey, "non-destructive quality grading of mango (mangifera indica 1.) Using image processing”, A dissertation submitted to Uka Tarsadia University, May 2014.

[25] Sergio Cubero et al., "Advances in Machine Vision Applications for Automatic Inspection and Quality Evaluation of Fruits and Vegetables", doi 10.1007/s11947-010-0411-8, Food Bioprocess Technol 4:487-504, 2011.

[26] Ghulam Muhammad, "Date fruits classification using texture descriptors and shape-size features", Engineering Applications of Artificial Intelligence, 37, 361-367, 2015.

[27] Sapan Naik and Bankim Patel, "Thermal imaging with fuzzy classifier for maturity and size based nondestructive Mango (Mangifera Indica L.) grading”, 2017 International Conference on Emerging Trends \& Innovation in ICT (ICEI), 978-1-5090-3404-8/17, 2017.

[28] Katrin Utai et al.,"Development and assessment of different modeling approaches for size-mass estimation of mango fruits (Mangifera indica L., cv. 'Nam Dokmai')", Computers and Electronics in Agriculture, 114, 269-276, May 2015.

[29] Longsheng Fu et al., "Classification of Kiwifruit Grades Based on Fruit Shape Using a Single Camera", Sensors (Basel), 16(7): 1012, doi: 10.3390/s16071012, July 2016.

[30] Moreda, G. P., et al. "Shape determination of horticultural produce using two dimensional computer vision-A review." Journal of Food Engineering 108.2: 245-261, 2012

[31] Kyosuke Yamamoto et al., "Strawberry cultivar identification and quality evaluation on the basis of multiple fruit appearance features", Computers and Electronics in Agriculture, Volume 110, Pages 233-240, January 2015.

[32] Muhammad, Ghulam. "Date fruits classification using texture descriptors and shape-size features." Engineering Applications of Artificial Intelligence 37: 361-367, 2015.

[33] Yudong Zhang et al., "Fruit classification using computer vision and feedforward neural network", Journal of Food Engineering Volume 143, Pages 167177, December 2014.

[34] Suchitra A. Khoje and S. K. Bodhe, "A Comprehensive Survey of Fruit Grading Systems for Tropical Fruits of Maharashtra", Critical Reviews in Food Science and Nutrition Vol. 55 , Iss. 12, 2015.

[35] F.S.A. Sa'ad et al.," Shape and weight grading of mangoes using visible imaging", Computers and Electronics in Agriculture, Volume 115, Pages 51-56, July 2015.

[36] Farid Garcia et al., "Fruit Classification by Extracting Color Chromaticity, Shape and Texture Features: Towards an Application for Supermarkets", IEEE Latin America Transactions, Volume: 14, Issue: 7, July 2016.
[37] Yudong Zhang et al.," Fruit classification by biogeography-based optimization and feedforward neural network", Expert Systems, Volume 33, Issue 3, Pages 239-253, June 2016

[38] Misigo Ronald et al., "Classification of selected apple fruit varieties using naive bayes", Indian Journal of Computer Science and Engineering (IJCSE), 2016.

[39] Girshick, R., Donahue, J., Darrell, T., et al., Rich feature hierarchies for accurate object detection and semantic segmentation. Comput. Sci., 580-587, 2014.

[40] Everingham, M.; Eslami, S.M.A.; van Gool, L.; Williams, C.K.I.; Winn, J.; Zisserman, A. The pascal visual object classes challenge: A retrospective. Int. J. Comput. Vis., 111, 98-136, 2015.

[41] Inkyu Sa et al.," DeepFruits: A Fruit Detection System Using Deep Neural Networks", Sensors, 16, 1222; doi:10.3390/s16081222, 2016.

[42] Alwanin, Rawabi. An Approach to Image Classification Based on SURF Descriptors and Colour Histograms. Diss. University of Manchester, 2014.

[43] Konaje, Nayan Kumar. "Food recognition and calorie extraction using Bag-of-SURF and Spatial Pyramid Matching methods."

[44] Chaivivatrakul, Supawadee, and Matthew N. Dailey. "Texture-based fruit detection." Precision Agriculture 15.6:662-683, 2014.

[45] Yogesh and A. K. Dubey, "Fruit defect detection based on speeded up robust feature technique," 2016 5th International Conference on Reliability, Infocom Technologies and Optimization (Trends and Future Directions) (ICRITO), Noida, pp. 590-594, 2016.

[46] Histogram of Oriented Gradients http://www.learnopencv.com/histogram-of-orientedgradients/. Date:20.06.2017.

[47] Tsolakidis, Dimitris G., Dimitrios I. Kosmopoulos, and George Papadourakis. "Plant leaf recognition using Zernike moments and histogram of oriented gradients." Hellenic Conference on Artificial Intelligence. Springer International Publishing, 2014.

[48] Southgate, Jamie. "Food Classification to provide Nutritional Information." 2016.

[49] Olsen, Alex, et al. "In Situ Leaf Classification Using Histograms of Oriented Gradients." Digital Image Computing: Techniques and Applications (DICTA), 2015 International Conference on. IEEE, 2015.

[50] http://www.pyimagesearch.com/2015/12/07/local-binarypatterns-with-python-opencv/. Date:20.06.2017.

[51] Bianco, Simone, et al., eds. Computational Color Imaging: 6th International Workshop, CCIW 2017, Milan, Italy, March 29-31, 2017, Proceedings. Vol. 10213. Springer, 2017.

[52] Tao, Huawei, et al. "Fruits and vegetables recognition based on color and texture features." Transactions of the Chinese Society of Agricultural Engineering 30.16: 305 311, 2014.

[53] Machine Learning A-ZTM: Hands-On Python \& R In Data Science by Udemy, 2017. 
[54] Woo Chaw Seng and Seyed Hadi Mirisaee, "A New Method for Fruits Recognition System," Electrical Engineering and Informatics, vol. 01, pp. 130-134, August 2009.

[55] Diego Sebastián Pérez et al., "Image classification for detection of winter grapevine buds in natural conditions using scale-invariant features transform, bag of features and support vector machines", Computers and Electronics in Agriculture 135, 81-95, 2017.

[56] Gonzalo Pajares, Xavier P. Burgos-Artizzu, Angela Ribeiro Alberto Tellaeche, "A computer vision approach for weeds identification through Support Vector Machine," Applied Soft Computing, pp. 908-915, 2011.

[57] Hong Zheng and Hongfei Lu, "A least-squares support vector machine (LS-SVM) based on fractal analysis and CIELab parameters for the detection of browning degree on mango (Mangifera indica L.)," Computers and Electronics in Agriculture, vol. 83, pp. 47-51, January 2012.

[58] Yifei Xu, Li Li , Xiaoli Li, Yong He Shuiguang Deng, "A feature-selection algorithm based on Support Vector Machine-Multiclass for hyperspectral visible spectral analysis," Journal of Food Engineering, May 2013.

[59] N.A. Semary et al., "Fruit-Based Tomato Grading System Using Features Fusion and Support Vector Machine",Advances in Intelligent Systems and Computing 323, DOI: 10.1007/978-3-319-11310-4_35, Springer International Publishing Switzerland 2015

[60] What are the various types of neural networks? https://www.quora.com/What-are-the-various-types-ofneural-networks.Date:16.06.2017.

[61] Ashwini Awate et al., "Fruit disease detection using color, texture analysis and ANN", Green Computing and Internet of Things (ICGCIoT), 2015 International Conference on, 10.1109/ICGCIoT.2015.7380603, IEEE, January 2016.

[62] O.O. Arjenaki, P. A.Moghaddam and A.M. Motlagh "Online tomato sorting based on shape, maturity, size, and surface defects using machine vision,\|. Turkish Journal of Agriculture and Forestry, vol.37, pp. 6268,2013 .
[63] M. T. Chowdhury, M. S. Alam, M. A. Hasan and M. I. Khan, Vegetables detection from the glossary shop for the blind, IOSR J. Electr. Electron. Eng. 8, 43-53, 2013.

[64] Fan et al., "Prediction of texture characteristics from extrusion food surface images using a computer vision system and artificial neural networks", Journal of Food Engineering 118(4):426-433 - October 2013 .

[65] Giacomo Capizzi et al.,"Automatic Classification of Fruit Defects based on Co-Occurrence Matrix and Neural Networks",Proceedings of the Federated Conference on Computer Science and Information Systems pp. 861867, DOI: 10.15439/2015F258, ACSIS, Vol. 5, 978-8360810-66-8, IEEE, 2015.

[66] Yudong Zhang et al, "Fruit classification using computer vision and feedforward neural network", Journal of Food Engineering 143:167-177 · December 2014.

[67] TensorFlow Tutorials https://www.tensorflow.org/tutorials. Date:17.06.2017.

[68] Google Trends: https://trends.google.co.in/trends/. Date:17.06.2017.

[69] Le, Q.V., 2013. Building high-level features using large scale unsupervised learning. In: Acoustics, IEEE International Conference on Speech and Signal Processing, Vancouver, pp. 8595-8598.

[70] JingLei Tang et al., "Weed identification based on Kmeans feature learning combined with convolutional neural network", Computers and Electronics in Agriculture 135 (2017) 63-70, January 2017.

[71] JingLei Tang et al., "Weed identification based on Kmeans feature learning combined with convolutional neural network", Computers and Electronics in Agriculture 135, 63-70, 2017.

[72] Train your own image classifier with Inception in TensorFlow https://research.googleblog.com/2016/03/ train-your-own-image-classifier-with.html?m=1, Date:16.06.2017.

[73] Krizhevsky, A., Sutskever, I., Hinton, G.E., ImageNet classification with deep convolutional neural networks. Adv. Neural Inform. Process. Syst. 25 (2), 1097-1105, 2012. 